\title{
Teaching Scientific Computing
}

\author{
B.A. Shadwick ${ }^{1,2}$ \\ 1 Institute for Advanced Physics \\ 10875 US Hwy. 285, Suite 199, Conifer, C0 80433 USA \\ 2 Center for Beam Physics \\ LBNL, Berkeley, CA 94720, USA \\ BAShadwick@IAPhysics.org
}

\begin{abstract}
We consider the wide scale use of computers in scientific research and the implications for the educational curriculum. We examine the practice of scientific computing from the perspective of obtaining robust numerical results. We explore the philosophy underlying this process and isolate the essential elements. We discuss in detail practices that are designed to minimize the likelihood of errors and also survey a set of supporting skills. Throughout, examples are used to illustrate the key ideas.
\end{abstract}

\section{Introduction}

Less than a generation ago, the routine use of computers in scientific research was largely restricted to specialists. CPU time was metered and in reasonably tight supply for all but the largest institutions. Modern tools and the tremendous computing power 1 that is routinely available to researchers has resulted in widespread, almost casual, use of computing in the physical sciences. A greater proportion of scientists are part-time practitioners of numerical computing, and numerous software packages make it seemingly easy to the casual user to do elementary numerical computations: to evaluate functions for graphing or for other purposes; to numerically (or symbolically) evaluate integrals; to solve ODEs and simple PDEs and so forth. Regrettably many of these scientists have little understanding of the mechanics underlying these numerical results. The scale of problem that the non-specialist is willing to take on scales with the growth in desktop computing power. This trend began with the general availability of desktop systems some two decades ago. It exists as a consequence of the natural human tendency to categorize; we make the (artificial) distinction between what we do on our desktop computers and what "computational physicists" do with supercomputers. We tend to view users of truly large-scale computing as specialists who have the detailed knowledge to perform these grand calculations, whereas the researcher who does the occasional computation using the a computer which is also used for email and writing papers does not need (or often even

\footnotetext{
${ }^{1}$ Of course, a college freshman might not consider today's computers necessarily that remarkable. Context is important.
} 
want) such specialized knowledge. The code that runs today in a few minutes on a workstation would have taken hours (if not days) on the central systems commonly in use twenty-five years ago. If we cannot distinguish specialists from non-specialists through the kinds of problems the address, then how can we differentiate them? Of course, there is no distinction and herein lies the potential problem.

As in society at large, scientists have come to view computers as mere appliances. Fortunately, it is also becoming more common for graduate and undergraduate programs to include courses in numerical methods. At the same time there is still a perception (probably less so in engineering than in, say, physics) that only students who plan to specialize in numerical work need to take such courses.

It is increasingly common for students to take on "large" computational projects with less-than-adequate supervision. Most faculty members would be reluctant to suggest a student tackle an analytical problem in an area where the advisor has little or no experience. Unfortunately, this same reticence does not extend to suggesting a student undertake numerical work, even when neither the advisor nor the student has significant computational experience. While there is something to be said for the "sink or swim" philosophy, failing to recognize the inherent complexity and subtlety of obtaining correct computational results does a grave disservice to students.

These observations argue that all students need more than a cursory introduction to numerical methods. Though impractical, computational methods should ideally be viewed as a mathematical tool as important as calculus, and receive similar weight in the curriculum. There is, for lack of a better term, a core process (and an accompanying mindset) by which we obtain computational results that we can believe. By concentrating on this crucial core, it is still possible to construct a course of study which the curriculum will bear.

We begin with a detailed examination of these processes, while providing examples which illustrate the key ideas. We turn next to broad survey of some essential skills and specialized knowledge required. By the end, we will have identified the essential elements, and hopefully have outlined a means to impart our process and our mindset to our students.

\section{Getting the Right Answer}

In this section we will examine the process by which we can obtain computational results in which we have confidence. To provide a reference, the discussion will be geared towards the task of obtaining numerical solutions of differential equations. Partial differential equations are ubiquitous in the physical sciences and students are often faced with formidable PDEs from which they must wrest a numerical solution. While the philosophy presented here is generic to scientific computing; some of the details will be different when considering numerical tasks other than solving differential equations. 
Perhaps this section could be more accurately titled "Avoiding Wrong Answers" as that is actually what one does. It is quite rare for one to be able to claim that a numerical calculation is "right," since such a claim would imply an independent, exact (i.e., an analytical) solution to the problem in question. If such a solution exists, then why the recourse to numerical solutions? So what do we meant by "the right answer"? The best we can normally accomplish is a belief that our computer code is not wrong. That is, at best, we find no evidence that the result of our calculation is wrong; we have successfully performed a series of tests - typically running the code with sets of parameters for which something is known about the solution (more on this below) — which show that the code is functioning as we expect. We then extrapolate this ostensively correct behaviour to cases where we know less about (but have more interest in) the solution. This is what we mean by our use of the term "right."

We do not wish to be overly pessimistic about computational results, but it is essential that we are mindful of the nature of the endeavour. In some respects, numerical computation has more in common with experimental science (in terms of assessing the outcome) that it does with analytical worl 2 . While numerical work is "theoretical," the required mindset more closely matches that of the experimentalist than the theoretician. An analytical calculation always "works"; one either finishes the calculation, or reaches a point where further progress appears impossible. The point where difficulties arise is clear and the calculator is typically aware of the step which serves as the stumbling block. An experiment, however, has a different character. In some cases, even though the apparatus is functioning as the experimentalist expects, there is no signal, i.e., the experiment doesn't work. Even when there is a signal, the experimentalist must determine whether this signal has the meaning intended when the experiment was designed.

The only demonstrable evidence that something is badly wrong with a numerical calculation occurs when the code crashes (i.e., the computer operating system terminates the program due to bad behaviour) or the output contains some quantity of NaNs. Absent these difficulties, some output is always obtained. Thus we are always faced with the question "Does the output of this code mean what we think it means?" The importance of recognizing this question is inherent in all numerical computation and cannot be over emphasised. We are always at risk of being misled by the output of our codes. There is a natural predisposition to believe all of those digits before us have some intrinsic meaning. Compiler errors and errors in the internal implementation of the machine instructions are thankfully quite rare 3 The output of a computer program is almost certainly in

\footnotetext{
2 The dreadful term "numerical experiment" should not come to mind in this context. Numerical computations are no more or no less than analytical theory; in both cases, the goal is to determine the predictions of a particular model. The term "numerical experiment" seems intended to elevate the stature of computing beyond that of other theoretical tools. Numerical "experiments" are not experiments. Unlike a real experiment, which always entertains the possibility of the discovery of new physical phenomena beyond what is already known, the output of a numerical computation can contain no more physics than the input.

3 The infamous Pentium division bug of the mid 1990's notwithstanding.
} 
precise agreement with what the programmer instructed (or thought he instructed) the computer to calculate. It is easy, especially for beginning practitioners, to confuse this correctness with the question of whether or not the algorithm as implemented (or as envisioned) does indeed solve the problem under consideration, given the current set of computational parameters. As Acton points out [1], most errors in computational results are ultimately due to human errors. Only by recognizing this fact can we instill practices and attitudes which will work to mitigate such errors.

We minimize the likelihood of errors by performing tests which indicate whether the numerical solution is consistent with the solution of the problem at hand. We will now discuss some techniques for building confidence in the results of a computation, as well as some general guidelines for avoiding problems.

Validation. The construction of effective tests is critical to verify proper operation of numerical codes. There are two classes of tests: those designed to test that the algorithm implemented in the code solves the problem it was designed to solve (referred to as benchmarks), and those designed to test the implementation logic (this class of tests is often referred to as regression tests by software engineers). While both classes of tests overlap in terms of the functionality they probe, the philosophy behind the two classes is markedly different.

Regression tests are primarily used to verify that alterations to a program do not result in unintended consequences. Regression tests are used to identify changes in behaviour over time; their purpose is to verify that a given set of inputs produces the same outputs today as yesterday. The cases used for regression testing do not have to produce meaningful output, only consistent output. The parameters do not need to be physically reasonable, nor does the resolution need to be fine enough to provide converged results. Consequently, regression tests can be very short runs that take small amounts of CPU time. Due to their nature, regression tests cannot be used to validate a code in an absolute sense. After a particular version of a code has been validated with benchmarks, the regression tests are run, the output is deemed to be correct, and these test results are then used for future comparisons. It is not uncommon for even moderate-sized codes to be run through a nightly set of regression tests. In any event, after code modifications, regression testing should be performed before any "production runs" are made.

Benchmarks, on the other hand, are meant to verify that a program solves its intended problem. This is normally accomplished by considering parameter regimes (e.g., small amplitude excitation) where either exact or approximate solutions exist, or where specific properties of the solution are known. For example, a linearized version of the model equations may be exactly soluble or parameters (or initial conditions) may exist which effectively reduce the dimensionality of the problem. The most valuable tests are those where an exact solution exists in a certain limit; these tests tend to be quite sensitive to algorithmic errors. Additionally, there are often analytical constraints on the solution (for example conservation of energy) which should be respected by the numerical solution to 
a level consistent with the algorithm. Generally, numerical solutions should be tested for agreement with all known analytical properties.

Recently, we encountered a rather serious example of poor benchmarking. There is a simple analytical relationship between the values of put and call options (so called put-call parity), yet we discovered that the software for computing options prices used by a trading desk in an investment bank produced results that significantly violated this constraint. This was a production setting: the software was used for negotiating contracts and to determine prices both for internal auditing purposes. An error of this nature should never have gone undetected by any reasonable testing procedure.

Convergence. Verifying convergence of the numerical solution is critically important; except for certain classes of ODEs and linear PDEs, there is no general theorem regarding the convergence of the solution of finite difference equations as the discretization parameters tend to zero 4 While there is no guarantee that the numerical solution will converge to the analytical solution (assuming such exists), a necessary condition for the numerical solution to be consistent with the analytical solution is that the numerical solution tends to a limit as the finite difference cell size tends to zero. Moreover, verifying that the convergence rate is consistent with the order of the discretization is a valuable test of a code and can uncover subtle errors. The most important reason for studying convergence is as a means to determine the resolution necessary to produce results of acceptable accuracy. It is quite difficult to determine, a priori, the necessary resolution. An example of this presented itself recently in our work related to the propagation of an intense laser pulse in a long plasma. One would normally suppose that a resolution of, say, ten to twenty grid-points per laser cycle would be sufficient to obtain reasonable results. Studying the convergence of the solution led to the surprising discovery that a resolution on the order of one hundred grid-points per cycle was necessary to obtain the correct results. At lower resolutions, the solution appeared quite reasonable, but in fact, the laser was coupling too strongly to the plasma and transferring energy considerably more rapidly than was physically correct. While the answer appeared plausible, it contained a qualitative error: the graph of laser energy versus time had the wrong concavity. This error was only uncovered by performing a series of runs that essentially produced a fully converged result. Often one hears comments to the effect "I doubled the number of grid-points and the solution only changed by ten percent, so that's good enough." While the asymptotic order of the error terms will be known from formal analysis, convergence rates will only match asymptotic estimates for sufficiently "small" grid sizes. Only by examining the changes in the solution over a series of step sizes is it possible to determine if the step sizes involved are such that the asymptotic error scaling applies. Furthermore, knowing that asymptotic scaling applies, in conjunction with observed convergence rates, allows for global error estimates.

\footnotetext{
${ }^{4}$ Clearly such a theorem would imply an existence theorem for the solution which explains the dearth of results for nonlinear PDEs.
} 
Algorithms. It is an exceedingly bad idea to modify the model equations solely for the convenience of numerical solution (an exception to this injunction is the conversion of a PDE to integral form from which a weak solution is obtained). For the most part, simplifications which appeal to the analytical eye do not necessarily make the numerical solution "easier" to obtain. Arithmetical complexity is not generally an important concern. It is certainly of questionable worth to reduce the validity of the model (say by linearizing certain terms) to "make it simpler for the computer." The complexity of the calculation is only at issue as it relates to amount of CPU time used to obtain a solution. Rarely is "making the equation easier to program" a valid consideration.5 In any case, it makes the most sense to implement the full model. After that code is properly working one can explore any "simplifications" which may prove advantageous. Experience suggests that it is universally easier to impose new assumptions than to remove existing ones.

As a general principle, there must always exist a control parameter (which is part of the input data) that in some limit takes the discretized equations into formal agreement with the model equations. Grid sizes and time steps introduced in the finite difference solutions of differential equations are examples of such control parameters. As an illustration of the danger of uncontrolled approximations, consider the following: the wave equation for a laser pulse propagating in a plasma was modified to facilitate solution, and the essence of the modification was that the formal expression for an inverse of the differential operator was approximated in Fourier space by expansion in a power series. Only if all terms were kept was there formal agreement with the unmodified equations. The code in question kept only the first term in this expansion, with the result that the group velocity of the laser pulse, after a long propagation distance, was grossly incorrect (as was the final spatial position of the pulse). In the absence of a control parameter, there was no easy way to discover that the program was, in fact, solving a very different equation than the author of the program intended. Provided the propagation distance was small enough, this error could be considered tolerable. Of course, as is virtually always the case, the original restriction on propagation distance imposed by this modification was forgotten, and the code was used in a regime where it was invalid. This discrepancy was only found when an independent group compared the (published) erroneous solutions with the results from a code which made no such approximations. It is worth noting that reliable algorithms for solving the unmodified equations were readily available, but apparently unknown to the researchers. (In the final analysis, in addition to producing the wrong answer, the modifications in question made the equations harder to solve numerically.) Modification of model equations should not be thought of as a numerical technique; there are likely assumptions about the solution and/or input parameters which are necessary for solutions of the modified model to approximate solutions of the original model. Above all, modifying an equation should be considered a last, desperate, resort.

\footnotetext{
${ }^{5}$ With the use of various code generation techniques, arguably, simplicity of implementation is never a valid consideration.
} 


\section{General Knowledge}

In addition to the techniques discussed in the previous sections, there also exists a general body of knowledge and skills with which researchers involved in computation must have at least a passing acquaintance.

Numerical Analysis. General numerical analysis is well covered in any number of references. The texts by Acton [1]2 are both eminently readable and genuinely insightful. They serve as an excellent starting point for acquiring an understanding of floating point arithmetic, round off error, numerical stability of algorithms, numerical integration, solving linear and non-linear equations and the like. This said, students must be aware that the "cookbook" approach to problem solving will inevitably fail. For example, any one of a number of "standard" methods can be used to solve a set of ODEs (assuming, of course, that there are not critical properties which must be exactly mirrored in the numerical solution), but with the exception of certain standard PDEs, off-the-shelf PDE solvers simply don't exist. Algorithms have to be constructed specifically for the equation in question.

To be successful, it is necessary to understand the mathematical basis for the various algorithms one uses. That all algorithms solve some problem exactly is an important realization. In the case of differential equations, one obtains numerically the exact solution of a slightly modified differential operator. This modified operator reduces to the original equations as the time-step and/or grid spacing tends to zero. In the case of systems of linear equations [3] the numerical result is a solution to a system with a slightly modified coefficient matrix. In all cases studying the modified equations can lead to significant insight into characteristics of the numerical solution. These techniques, collectively known as backwards error analysis, are the bases for constructing the structure preserving methods.

Software Engineering. There has been much improvement in software engineering practices in recent years. Ideally, practitioners of scientific computing should, as much as possible, avail themselves of the current "best practices" in software engineering. While not wanting to wade into the partisan arguments over which programming language is best suited to scientific computing, it is nonetheless worth noting that object-oriented design and coding practices now dominate commercial software development and for very good reason. Adhering to good design principles (encapsulation, abstraction, structured programming, etc.) can greatly reduce the possibility of bugs as well as improve productivity. Regardless of which language is used, it is critical to have a thorough understanding of how to properly use the chosen language. In particular, it is important to understand that most languages will have undefined behaviours - language constructs which will compile but whose consequence at runtime is not defined by the language standard - any of which can result in hard-to-find bugs, and therefore, must be actively avoided.

In addition to understanding the proper use of the programming language, the use of some form of source code revision control is also essential. A fun- 
damental aspect of science is the reproducibility of results. All to often, this hallmark is missing from computational work. Research codes are constantly changing, and only through tracking source code changes is it possible to revisit previous work. A critical element of this reproducibility is the ability to know which version of the code produced a particular result. For example, each time one of our own codes is run, the parameters, along with the current version of the code, are stored and the run is assigned a unique identifier. In addition, all hard (and soft) copies of results are labeled with this same identifier. This identifier, in conjunction with version control, allows any run (especially those used in publications) to be reproduced at any time.

\section{Conclusions}

Few students will master all the material we have discussed here during their graduate careers, nor should we expect this to occur. This material should be regarded as general framework that we encourage our students to fill in as appropriate. The question of what should comprise the undergraduate curriculum is particularly challenging, given all the forces competing for lecture time. At the undergraduate level, beyond the usual elementary numerical analysis, it is most important to emphasize the difficulty of validating any computation. This mindset is critical; if we can condition our students to ask "is this right?" then they will experience much greater success, while simultaneously encountering less frustration.

Acknowledgements. The advice contained is this paper is the consequence of over two decades of practical computing. Over that time, the author has benefited from countless discussions about software and numerical methods with colleagues and collaborators alike and with pleasure acknowledges John C. Bowman, W. F. Buell, Andrew Charman, E. H. Esarey, Richard Fitzpatrick, C. B. Schroeder, W. F. Shadwick, J. D. Talman, and G. M. Tarkenton. This work was supported by the US DoE under contract No. DE-AC03-76SF0098 and by The Institute for Advanced Physics.

\section{References}

1. Acton, F.S.: REAL Computing Made Real: Preventing Errors in Scientific and Engineering Calculations. Princeton University Press, Princeton, NJ (1996)

2. Acton, F.S.: Numerical Methods That Work. Mathematical Association of America, Washington DC (1990)

3. von Neumann, J., Goldstine, H.H.: Numerical inverting of matrices of high order. Bull. Amer. Math. Soc. 53 (1947) 1021-1099

4. Turing, A.M.: Rounding-off errors in matrix processes. Quart. J. Mech. Appl. Math. 1 (1948) 287-308 